\title{
A QoS Routing Protocol for Mobile Ad Hoc Networks Based on a Reservation Pool
}

\author{
Donghak Pyo ${ }^{1}$, Sunggu Lee ${ }^{1}$, and Min-Gu Lee ${ }^{2}$ \\ ${ }^{1}$ Electrical and Computer Engineering Division \\ Pohang University of Science and Technology (POSTECH) \\ San 31 Hyoja Dong, Pohang, Korea \\ \{dhpyo, slee\} @postech.ac.kr \\ ${ }^{2}$ Network Control Platform Development Team \\ $\mathrm{BcN}$ Business Unit, Korea Telecom Corporation, Seoul, Korea \\ bluehopeakt.co.kr
}

\begin{abstract}
Even without possible interference from external radio sources, the problem of guaranteeing quality-of-service $(\mathrm{QoS})$ routing in mobile ad hoc networks (MANETs) is a difficult problem. Difficulties arise because node mobility can cause frequent network topology changes, communication channels can have high error rates, the jitter rate is high and several different applications can be sharing the use of the communication medium. This paper addresses these issues and proposes a new QoS routing protocol for MANETs that combines aspects of a MAC protocol and a path reservation protocol. The proposed QoS routing protocol can be implemented based on any routing method that supports multiple paths (e.g., DSDV, TORA, PDR). The QoS performance of the proposed protocol is verified with simulations conducted using NS-2.
\end{abstract}

Keywords: Mobile ad hoc network (MANET), quality-of-service (QoS), routing, time division multiple access (TDMA).

\section{Introduction}

There are many interesting applications that can be supported if quality-of-service (QoS) support can be provided for mobile ad hoc networks (MANETs). Such applications include reliable mobile multimedia services, disaster recovery and realtime identification of mobile objects in a battlefield environment. However, because MANETs typically use electromagnetic-wave-based wireless communication protocols, such as IEEE 802.11 or ZigBee, it can be difficult (or even impossible) to control possible interference from external radio sources. For example, if an ad hoc IEEE $802.11 \mathrm{~b} / \mathrm{g}$ network (named Network A) is created, then other electronic devices using Bluetooth (which uses the same $2.4 \mathrm{GHz}$ frequency range) or IEEE $802.11 \mathrm{~b}$ connections outside of Network A can interfere with communication within Network A. Thus, IEEE $802.11 \mathrm{~b} / \mathrm{g}$ uses a communication protocol based on carrier sense multiple access with collision avoidance (CSMA/CA), which is a protocol that 
inherently cannot guarantee real-time communication. Since, in general, possible interference from external radio sources cannot be controlled, exclusive reservation of communication bandwidth (for a particular application) cannot be achieved, and thus, complete quality-of-service (QoS) support cannot be achieved.

Given the fact that the behavior of external radio sources cannot be controlled, the best that can be hoped for in a MANET environment using wireless links is to create a communication protocol that prevents interference between any two communication channels that strictly adhere to the protocol created. There have been several previous attempts to create such a protocol. However, all previously proposed protocols have drawbacks that limit their usability. Thus, in this paper, a new QoS routing protocol is proposed for use in MANETs.

Let us assume that there is no interference from external radio sources. Even with this restriction, the problem of guaranteeing quality-of-service $(\mathrm{QoS})$ routing in mobile ad hoc networks (MANETs) is a difficult problem. For a given network, sufficient resources must be reserved in order to satisfy the QoS requirements of a particular application. However, in a MANET, node mobility can cause frequent network topology changes, communication channels can become unreliable and have high error rates, the total available bandwidth tends to be lower than in wired networks, the jitter rate is high and several different applications can be sharing the use of the communication medium. In this paper, we propose a new QoS routing protocol that combines aspects of a MAC protocol and a path reservation protocol. First, a MAC protocol based on a multihop TDMA method, adapted to a MANET environment, is used to permit exclusive reservation of network bandwidth resources. Second, a reservation pool method, in which network resources are reserved in advance and placed in a reservation pool, is used to deal with the problem of frequent network topology changes.

The rest of this paper is organized as follows. Section 2 provides an overview of previously proposed QoS routing protocols that can be applied to MANETs. Summaries of the proposed protocols and their drawbacks are described. Next, a new QoS routing protocol that overcomes these drawbacks is described in Section 3. Simulation results are presented in Section 4. Finally, the paper concludes with concluding remarks in Section 5.

\section{Related Work}

In [1], Lin and Liu proposed a QoS routing method for MANETs built on top of the Direct Sequence Distance Vector (DSDV) routing algorithm. To overcome a potential hidden terminal problem, Lin and Liu propose the use of a code division multiple access (CDMA) technique applied on top of a time division multiple access (TDMA) method. [1] proposes a systematic method for computing the link bandwidth and path bandwidth available to a virtual circuit. Code and time slot resources are reserved for primary and secondary paths that meet the pre-specified bandwidth constraints for a given virtual circuit request. If a link in the primary path becomes disconnected due to node movement or other reasons, the virtual circuit is switched to the secondary path. This method has the drawback of requiring the reservation of valuable network resources for a backup path that may not be used. In addition, the 
need to implement CDMA over TDMA results in a complex system that may not be appropriate for use in applications with low-performance processors such as wireless sensor networks.

The Ticket Based Probing (TBP) protocol is an alternative QoS-aware routing protocol proposed by Chen and Nahrstedt [2]. Tickets are used for arbitration - the holder of a ticket is given permission to search for a path. Two types of tickets are used: yellow and green. A yellow ticket indicates a preference for paths with shorter delays. A green ticket indicates a preference for lower-cost paths. Flow control is implemented by limiting the number of yellow and green tickets allocated to the network. A localized path repairing scheme is provided to deal with mobile nodes. Three levels of path redundancies are provided in TBP. In the first level of path redundancy, multiple routing paths are established for a single message stream (a separate copy of each data packet is sent only each path). With the second level of path redundancy, multiple routing paths are established, but data packets are only sent along one primary path. The other paths are backup paths that are only used if the primary path becomes disconnected. However, note that the presence of backup paths has an adverse affect on other message streams since valuable network resources have to remain reserved for the backup paths. In the third level of path redundancy, a secondary path is only established when the primary path fails. Such a method will result in a long recovery time - this may result in a temporary failure to meet prespecified QoS requirements.

A method referred to as "Stateless Wireless Ad hoc Networks (SWAN)" is also proposed as a QoS routing protocol for MANETs [3]. The term "stateless" in the name of this method refers to the fact that per-flow or aggregate state information does not need to be stored at each node. This method instead relies on feedback information obtained from the network. When a circuit needs to be established, a probe packet is sent towards the destination. Upon observing a probe packet, each intermediate node marks its bandwidth in the packet header if its own available bandwidth is detected as the bottleneck bandwidth. Upon receiving the probe packet, the destination node replies to the source node with the bottleneck bandwidth value. The source node initiates a rerouting procedure if the bottleneck bandwidth is insufficient to support the necessary level of QoS. This type of route discovery process may incur a large amount of control overhead and a long delay. In addition, false admission is possible because forward reservation is not being used.

In [4], Shih et. al. propose another TDMA-based routing protocol meant to support QoS routing in MANETs. This method, referred as a Distributed Slots Reservation Protocol (DSRP), is designed to be an improvement over Lin and Liu's method [1] based on using CDMA over TDMA. In order to obviate the need for implementing CDMA on top of TDMA, and thereby complicating the system implementation, reasoning about possible conflicts due to hidden terminal and exposed terminal problems is used to determine the set of time slots that can be used by a given node without fear of running into conflicts with other transmitting nodes. This reasoning is implemented in a set of algorithms executed at each node. Route maintenance is implemented by executing a new route discovery phase when a link breakage is detected. Due to this dynamic route maintenance mechanism, however, route recovery may take a long time and QoS requirements may not be met as a result. 


\section{Proposed QoS Routing Protocol}

The QoS routing protocol proposed in this paper overcomes the problems of high complexity, waste of network resources, and/or slow recovery from link breakage observed with the previously proposed MANET QoS routing protocols. There are two aspects to the proposed routing protocol. First, a multi-hop TDMA method is used to provide a simple method of allocating time slots to communication channels such that no two transmitting nodes conflict with each other. Second, a reservation pool method, based on [5], is used to deal with the problem of frequent network topology changes. The following subsections present the assumptions used in the proposed protocol and the above two aspects of this protocol.

\subsection{Assumptions}

Before delving into the details of the proposed method, let us briefly outline the assumptions being made in this paper. First, it is assumed that external radio interference, if any, will not adversely affect the real-time performance of the proposed routing protocol. Of course, since we cannot control the behavior of all external devices that emit radio waves, it is possible for a wireless communication channel to become disrupted for a short time interval. However, in such a case, we are assuming that appropriate forward error correction techniques can be used to automatically make corrections to the data as it is received at each intermediate node. If external radio interference is of a sufficiently long duration, then no routing protocol will be capable of providing real-time communication guarantees. Thus, we are simply assuming that such is not the case.

Second, a separate clock synchronization mechanism is assumed. Several such clock synchronization mechanisms, including the beacon-based method used in the IEEE 802.11 protocol, are available for use in MANET environments. A clock synchronization mechanism is necessary in order to use TDMA to coordinate the transmission and reception of packets over a wireless communication medium, which is of a broadcast nature by default. The granularity of the clock synchronization method used will determine the duration of the guard time required between adjacent time slots in our TDMA scheme.

Third, as in [1], the only QoS parameter of interest will be assumed to be the bandwidth available to a given virtual circuit between a source node and a destination node. Thus, other possible QoS parameters such as end-to-end latency, signal-tointerference ratio and packet loss rate are not considered in this paper. This assumption is made because bandwidth guarantees are the most critical factor for typical real-time applications. Consideration of other possible QoS parameters will be left as future work.

\subsection{Multihop TDMA}

Suppose that a given source node wishes to send a sequence of data packets, with certain QoS guarantees, to another node in the MANET. Then the source node can send out probe control packets in order to find a path to the destination node and to reserve appropriate network resources along that path. A set of reserved network 
channels from the source node to the destination node, using the path found, will form a virtual circuit that can be used to subsequently send out the data packets requiring QoS guarantees. A key requirement of this type of procedure is the capability of reserving network resources. If link bandwidth is considered as the primary network resource of interest, then a method is required to partition the total available bandwidth on a given link and to reserve portions of the total available link bandwidth. A natural way to achieve this is to use TDMA on that link. However, since end-to-end QoS guarantees will be required for the virtual circuit to be created, a multihop TDMA protocol will be required for a MANET environment.

Let us first consider the partitioning of the time available for communication on a single link of a given MANET. For this purpose, time can be partitioned into superframes, with a superframe partitioned as shown in Figure 1. Although this structure appears similar to the frame structure used in [1] and [3], it is actually slightly different because each slot in the control phase is allocated to a specific node in our network (there are as many control phase slots as there are nodes) and each slot in the data phase is used to partition the link bandwidth available for sending data packets. Thus, the idea is that a node can use the control phase slot allocated to it to request allocation of a data phase slot for its exclusive use when sending data packets to a specific neighboring node. Although such a method limits the size of the MANET that can be supported, it is nevertheless a simple and low-overhead method that can be used with appropriately-sized MANETs.

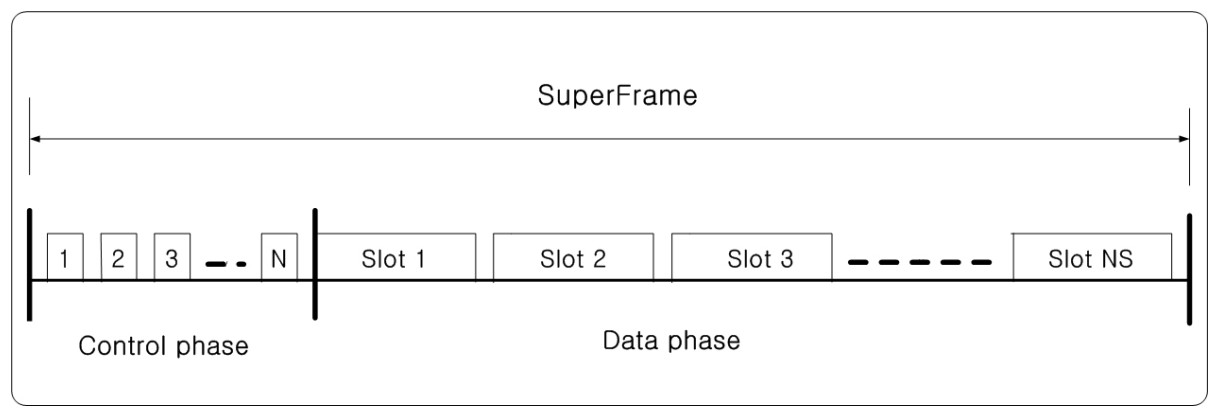

Fig. 1. The structure of a superframe used for bandwidth reservation on a single link

When using the above type of superframe structure, care must be taken to ensure that nodes that are two or fewer hops away from each other do not attempt to use the same data slot. Unless handled properly, such a conflict can result in a so-called "hidden terminal" problem, which is the reason that [1] uses CDMA on top of TDMA. To ensure that there are no potential conflicts with two different packets, received at the same time at an intermediate node, from two different nodes, slot usage information can be exchanged between neighboring nodes.

In particular, each node can maintain a two-dimensional slot-usage state table. This state table only needs to contain information about the data slots being used by neighbors and the neighbors of neighbors. Thus, each node informs its neighbors of the data slots that it has reserved. Those neighbors then pass on this information for one more hop. Figure 2 shows the format of the state table used with example data 
entries. Node mobility is taken into account by maintaining an "exists" bit that is set when a neighboring node moves into the radio range of the current node; the "exists" bit for a particular node is reset when that node moves out of the radio range of the current node. When making a data slot assignment request, a node only needs to refer to the data slot usage of those nodes with the "exists" bit set to one.

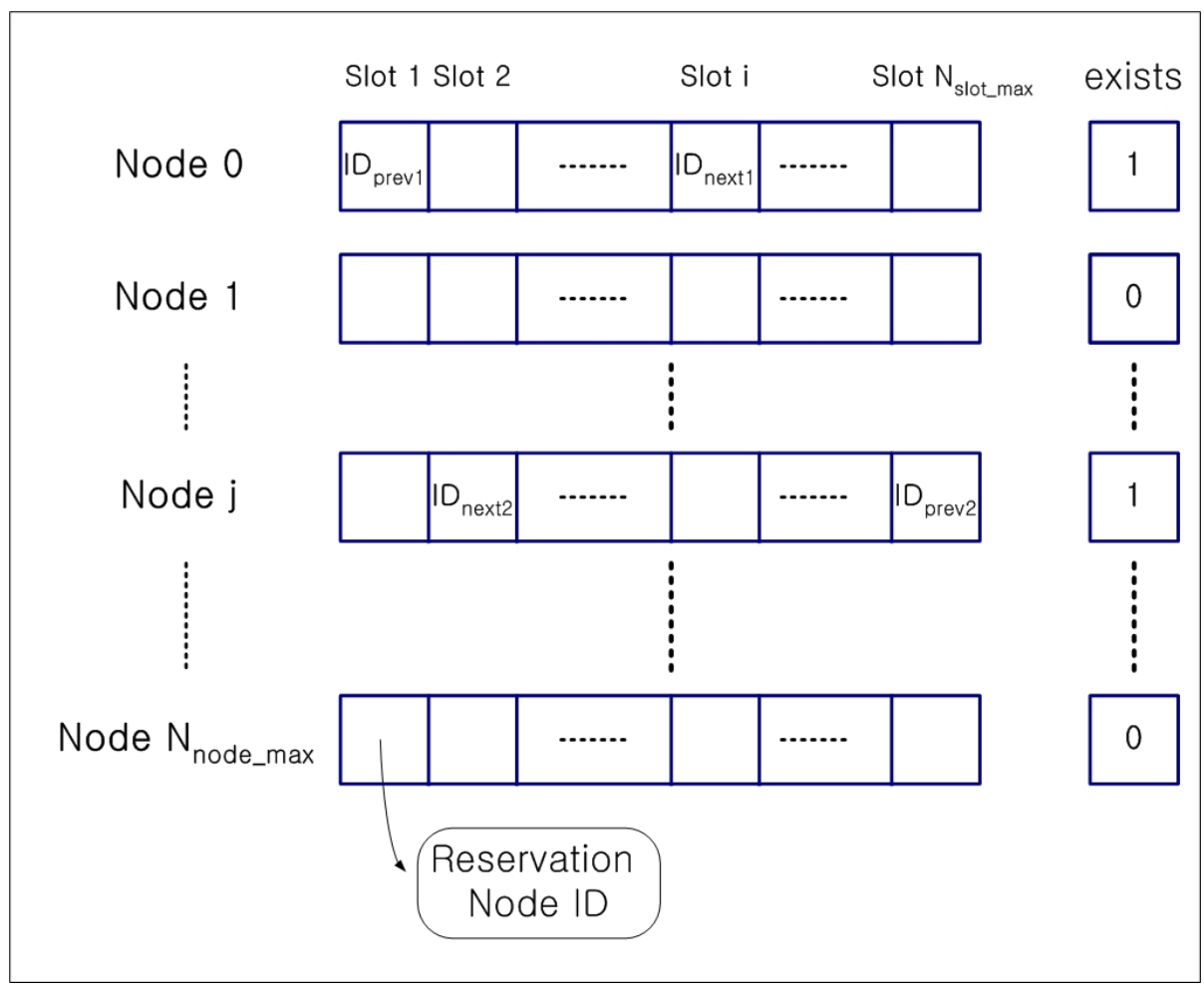

Fig. 2. The slot-usage state table and "exists" bit column with example data

By maintaining appropriate state information, of a manageable size, at each node, the time slots that can be utilized by a given node without conflicting with other nodes can be determined in a much simpler method than that used in [4]. In the example data shown in Figure 2, which could be a slot-usage state table stored at node 0, node 0 is receiving data from a node with ID number $\mathrm{ID}_{\text {prev1 }}$ using slot 1 and sending data to node $\mathrm{ID}_{\text {next1 }}$ using slot $\mathrm{i}$. Node $\mathrm{j}$, which is a neighboring node in the radio range of node 0 , is sending data to node $\mathrm{ID}_{\text {next2 }}$ using slot 2 and receiving data $\mathrm{ID}_{\text {prev2 }}$ using slot $\mathrm{N}_{\text {slot_max }}$. Since node 0 knows the slots being used by its neighboring nodes (this information is stored in its slot-usage table), node 0 can avoid those slots when reserving bandwidth for a new channel to another adjacent node. Thus, if node 0 wishes to communicate with node 1 after node 1 moves into the radio range of node 0 (which will result in the "exists" bit for node 1 being changed from a 0 to a 1 ), then it can reserve any slot except the slots $0,1, \mathrm{i}$ and $\mathrm{N}_{\text {slot_max }}$. 


\subsection{Reservation Pool Method}

Suppose that a virtual circuit is created by reserving network resources along a primary path. Data packets requiring QoS guarantees can be sent using such a virtual circuit. However, since a MANET environment is assumed, nodes can move around. Thus, in order to maintain a virtual circuit with the required QoS, path maintenance will be required to switch over to alternate paths or to repair the current primary path when link breakage occurs in the primary path. This problem was handled in different ways by the previous QoS methods surveyed in Section 2. However, as noted there, methods that reserve network resources for a backup path in advance can waste precious network resources if the backup path is not needed, while methods that search for a new path when the primary path breaks can require long path recovery times.

The solution proposed in this paper is to maintain a pool of network resources, reserved in advance and shared by all virtual circuits that may require such resources. If a potential link breakage in a primary path is detected (based on detection of current node movements and estimates of future node movements), path maintenance is initiated. This is done by searching the reservation pool for a set of channels (a channel is a link and its associated data slot reservation, which together creates a communication pathway with a guaranteed bandwidth) that can be used to form a detour path with the required level of QoS. If such a set of channels are found, those channels are removed from the reservation pool and the primary path is modified to use the detour path. If the channels for such a detour path are not found in the reservation pool, control packets are sent out to search for a detour path. In both cases, the primary path is modified to use the detour path before the link breakage occurs, if possible.

By using a reservation pool as described above, path recovery will be much quicker than if new network resources have to be found every time a link breakage occurs. Also, since the resources in the reservation pool can be shared, there is much less waste of precious network resources. This solution is based on a method previously presented by two of the authors in [5]. Potential link breakage can be detected using a novel link stability estimation model and method presented in [7]. That paper also discusses other link stability estimation methods presented in the literature. Interested readers are referred to these papers for details on these methods.

\section{Simulation Results}

In order to evaluate the performance of the proposed QoS routing protocol, computer simulations were conducted using the NS-2 (Version 2.26) simulator [8]. Although simulations were performed using various sets of parameters, in this section, results are only shown for a representative set of environment and protocol parameters since results for other sets of parameters were similar. The simulation environment considered consists of 30 mobile hosts roaming about in a $1500 * 500 \mathrm{~m}^{2}$ area according to the Random Waypoint model [8]. Hosts move around with a random uniform speed (with maximum speeds ranging from $0 \sim 20 \mathrm{~m} / \mathrm{s}$ ) and an initial randomly chosen direction (with random direction changes). Each mobile host has 
the same transmission range of $250 \mathrm{~m}$. A transmission rate of $2 \mathrm{Mbps}$ is used. The superframe used in the simulations consists of control and data phases as shown in Figure 1. The slot time for each frame in the control phase is set to $1.2 \mathrm{~ms}$ and the total number of slots in the control phase is set to 30 , which is also the total number of mobile hosts. The slot time of each frame in the data phase is set to $5 \mathrm{~ms}$ and the total number of slots in the data phase is set to 16 . Therefore, the total length of a superframe is $116 \mathrm{~ms}=(30 * 1.2)+(16 * 5)$. Pseudo Distance Routing (PDR) is used as the underlying routing protocol [5]. The source-destination pair for a "call" (a virtual circuit connection) is chosen according to a uniform random distribution. For each call, traffic is generated with bandwidth requirements of 1, 2 or 3 slots (denoted as QoS1, QoS2 and QoS3, respectively) chosen in a uniform random manner. The total simulation time is set to 250 seconds.

In the first set of experiments, we consider the call setup time and bandwidth as the metrics of performance for the proposed protocol. The call setup time is the time required to reserve all of the slots in a path to the destination. Figure 3 shows the simulation result for call setup time versus node mobility. We compare call setup time while varying the number of hops in the path. When the node mobility is low, the number of probing messages used by the routing protocol (PDR) is small. The queuing time before transmission of the control packet is short. However, when the node mobility increases, the number of probing messages is also increased. Thus, the call setup time also increases. As shown in Figure 3, call setup time increases in direction proportion to the number of hops in the path.

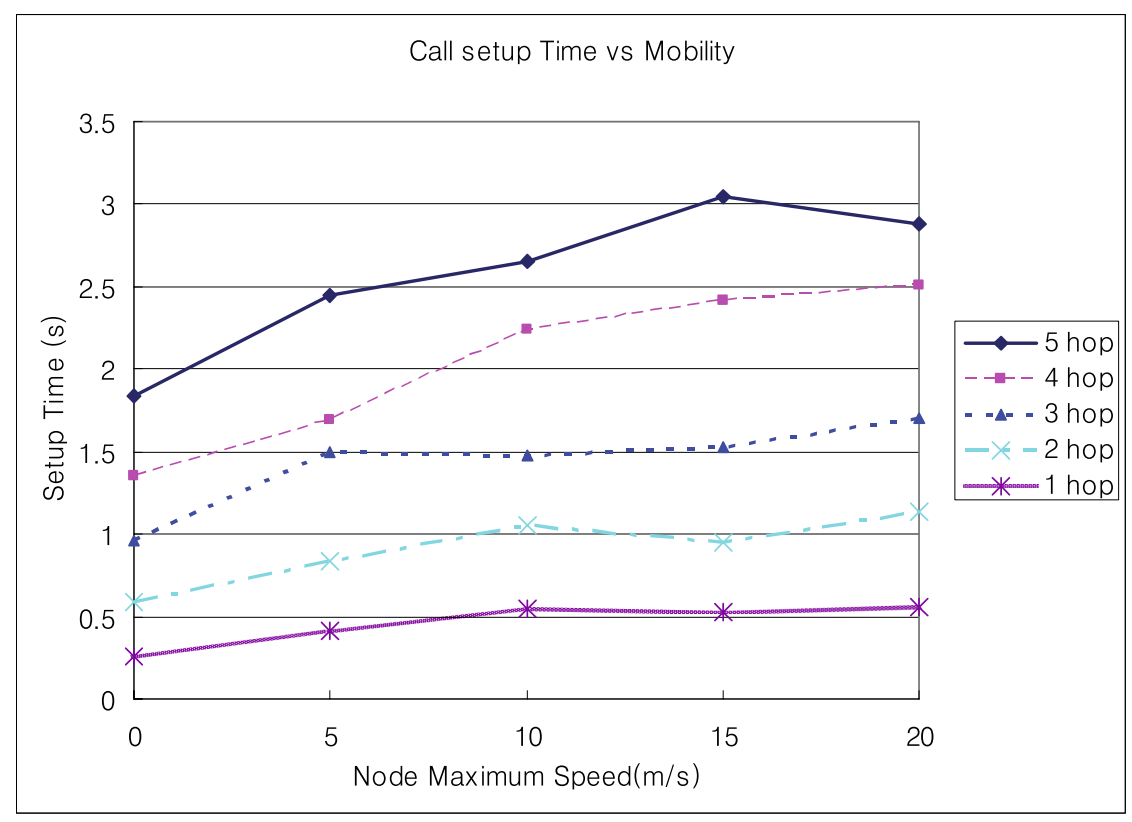

Fig. 3. Call Setup Time vs Mobility 
In the second set of experiments, the average throughput was measured for connections with different QoS requirements. As was done in [1], we assumed that each mobile host can request a connection to a destination node with different throughput requirements. The three types of throughput levels that can be requested, QoS1, QoS2 and QoS3, use 1, 2 and 3 slots, respectively, in the data phase of a superframe (refer to Figure 1). Each data packet size is assumed to be 1000 bytes long and the average interarrival time of packets is set to 30 milliseconds. If path reservation is successful, the source node sends 30 data packets to its destination. Figure 4 shows the throughput with various mobility levels. Overall, the average throughput of QoS1 is $67.0 \mathrm{Kbps}$, QoS2 is $125.5 \mathrm{Kbps}$ and Qos 3 is $183.7 \mathrm{Kbps}$. These simulation results show that the proposed QoS protocol can be used to provide different levels of throughput to circuits with different QoS flow requirements in a consistent, predictable manner.

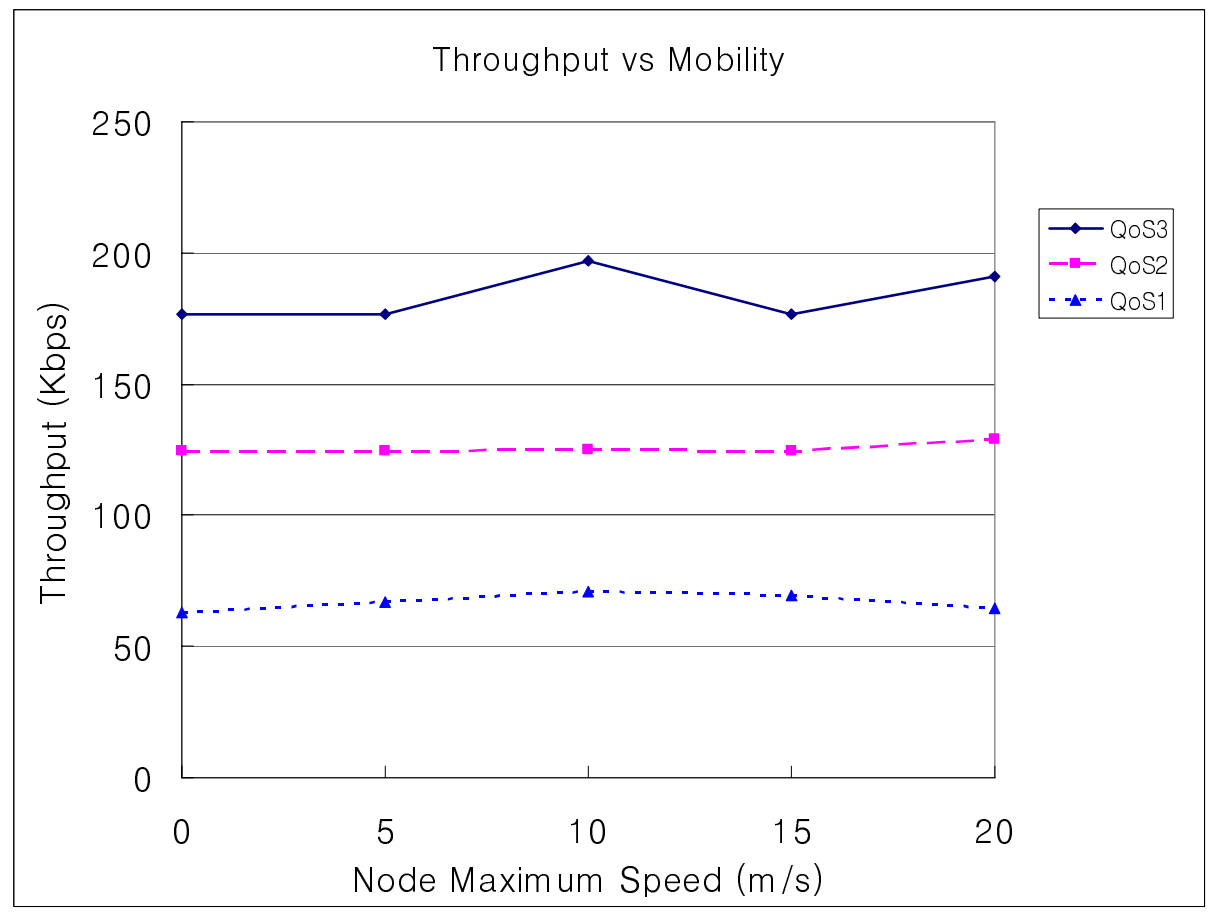

Fig. 4. Throughput vs Mobility

\section{Conclusion}

This paper has presented a new QoS routing protocol for MANET environments. It has been shown that all previously proposed QoS routing methods for MANETs have drawbacks that limit their usage. The QoS routing protocol presented in this paper gets around these drawbacks by using a multihop TDMA protocol and a reservation 
pool of network resources. The multihop TDMA protocol presented uses a superframe structure and a slot-usage table stored at each node in order to provide a simple method of allocating time slots to communication channels such that no two transmitting nodes conflict with each other. A backup reservation pool method is used to enable fast recovery when a primary path becomes disconnected because of links that break due to mobile nodes. The performance of the proposed QoS routing protocol is verified with NS-2 simulations. The simulation results show that this protocol can be used to provide different levels of throughput to circuits with different QoS requirements in a highly stable and predictable manner in MANET environments.

\section{References}

1. Lin, C.R., Liu, J.: QoS Routing in Ad HocWireless Networks. IEEE J. on Selected Areas in Communications 17(8), 1426-1438 (1999)

2. Chen, S., Nahrstedt, L.: Distributed Quality-of-Service Routing in Ad Hoc Networks. IEEE J. on Selected Areas in Communications 17(8), 1488-1505 (1999)

3. Ahn, G.-S., Campbell, A.T., Veres, A., Sun, L.-H.: Supporting Service Differentiation for Real-Time and Best-Effort Traffic in Stateless Wireless Ad Hoc Networks (SWAN). IEEE Trans. Mobile Computing 1(3), 192-207 (2002)

4. Shih, K.-P., Chang, C.Y., Chen, Y.-D., Chuang, T.-H.: A Distributed Slots Reservation Protocol for QoS Pouting on TDMA-based Mobile Ad Hoc Networks. In: ICON. Proc. 12th International Conference on Networks, vol. 2, pp. 660-664 (2004)

5. Lee, M.-G., Lee, S.: Pseudo-Distance Routing(PDR) Algorithm for Mobile Ad-hoc Networks. In: Proc. ITC-CSCC2005, vol. 2, pp. 797-798 (2005)

6. Lee, M.-G., Lee, S.: QoS Support for Mobile Ad-Hoc Networks Based on a Reservation Pool. In: ISORC. Proc. 9th IEEE International Symposium on Object and ComponentOriented Real-Time Distributed Computing, pp. 194-204. IEEE Computer Society Press, Los Alamitos (2006)

7. Lee, M.-G., Lee, S.: A Link Stability Model and Stable Routing for Mobile Ad-Hoc Networks. In: Sha, E., Han, S.-K., Xu, C.-Z., Kim, M.H., Yang, L.T., Xiao, B. (eds.) EUC 2006. LNCS, vol. 4096, pp. 904-913. Springer, Heidelberg (2006)

8. The network simulator, NS-2 Notes and documentation and source code. Available: http:// www.isi.edu/nsnam/ns/ 